\title{
On completeness of root vectors of Schrödinger operators: a spectral approach
}

\author{
Xiangdong Yang \\ Department of Mathematics, KunMing University of Science and Technology, 650093 KunMing, YunNan Province, China.
}

Communicated by S.-H. Rim

\begin{abstract}
We study complete properties of root vectors of Schrödinger operators. More accurately, denote by $B\left(r_{0}\right)$ be the ball centered at the origin with radius $r_{0}$ and $L^{1}\left(B\left(r_{0}\right)\right)$ the space which consists of real functions $f(x)$ satisfying $\int_{B\left(r_{0}\right)}|f(x)| d x<\infty$, then the complete properties of eigenvectors for Schrödinger equation are characterized. Our characterization depends on the sum of eigenvalues. Our proof is based on a complex-analytic conjugate approach which is widely used in the investigation of completeness of function systems in Banach spaces. (C)2017 All rights reserved.
\end{abstract}

Keywords: Schrödinger operators, inverse eigenvalue problem, completeness. 2010 MSC: 35A99, 30B60.

\section{Introduction and notations}

By a complete system of elements $\left\{e_{k}\right\}$ of a Banach space B, we mean $\overline{\operatorname{Span}}\left\{e_{k}\right\}=B$, i.e., the completeness is equivalent to the possibility of an arbitrary good approximation of any element of the space by linear combination of elements of this system. The completeness of solutions or root vectors of PDE and ODE has been investigated extensively. It is well-known that this problem is interesting both in mechanics and differential equations. As far as we know, the completeness of root vectors of ordinary differential equations could be traced back to 1970s, see [12] for example. The completeness problem of ODE is in connection with analysis (see, [7] and [13] ). In [16], the completeness of eigenfunctions for non-self-adjoint Sturm-Liuville operators are investigated by using the characteristic determinant. The completeness of the Floquet solutions are investigated for the parabolic equation which describes small oscillations of a fluid-system in [8].

Completeness of solutions of partial differential equations were considered in [9], where applications to inverse problems were also given. A uniqueness theorem was established for inverse scattering with fixed energy data in [14]. The phase shifts in this theorem are of some practical significance because they can be measured in some physical experiments. They are also related to the classical Müntz Theorem which states the necessary and sufficient conditions for a system of polynomials with gap to be complete

Email address: yangsddp@126. com (Xiangdong Yang)

doi:10.22436/jnsa.010.01.22 
in the Banach space consisting of continuous functions on a finite interval. In [3], the completeness of eigenvectors for elliptic operators is proven. The completeness problem for abstract elliptic equations is investigated in [17].

The completeness of the eigenmodes of a grounded parallel plate waveguide is given in [10], whose proof is based on a general theorem governing the completeness of sets of complex exponentials, which is similar to the complex-analytic approach to [18, 20] and [19].

Most of papers on completeness of Schrödinger operators are characterized by boundary conditions. In [2], the completeness of solution system is characterized by the characteristic root of the equation. For delay equation $y^{\prime}(t)=a y(t-1)$, denote by $\left\{\alpha_{n}\right\}$ the characteristic root of the equation, then the solution system becomes $\left\{e^{i \alpha_{n} t}\right\}$. The author reduces the investigation of properties of solution system to exponential system.

As is well-known, there has been a lot of activity in connection with spectra with Schrödinger operators (see [5] and [11] for example). Inspired by [2], in this paper, we will investigate the completeness of root vector systems associated to Schrödinger operators by spectral conditions rather than boundary conditions. Our proof is based on the complex-analytic approach which is similar to [10, 18, 20] and [19]. More accurately, we will consider PDE of the form

$$
\left(\Delta+\lambda^{2}-\mathrm{q}(x)\right) h=0,
$$

where $q(x)$ satisfies the following:

Assumption 1.1. Let $q$ be compactly supported, i.e., $q(x)=0$, for $|x|>r_{0}$, where $r_{0}>0$ is an arbitrary large fixed number; $q(x)$ is real-valued, i.e., $q=\bar{q}$; and $q(x) \in H_{0}^{k}\left(B_{r_{0}}\right), k>3$.

Throughout this paper, points of $\mathbb{C}^{n}$ will be denoted by $z=\left(z_{1}, \ldots, z_{n}\right)$, where $z_{k} \in \mathbb{C}$. If $z_{k}=x_{k}+i y_{k}$, $x=\left(x_{1}, \ldots, x_{n}\right), y=\left(y_{1}, \ldots, y_{n}\right)$, then we write $z=x+i y$. The vectors $x=\mathfrak{R} z$ and $y=\mathfrak{I} z$ are the real and imaginary parts of $z$, respectively, $\mathbb{R}^{n}$ will be thought of as the set of all $z \in \mathbb{C}^{n}$ with $\Im z=0$. The notations

$$
\begin{aligned}
|z| & =\left(\left|z_{1}\right|^{2}+\cdots+\left|z_{n}\right|^{2}\right)^{1 / 2}, \\
|\mathfrak{R} z| & =\left(\left|x_{1}\right|^{2}+\cdots+\left|x_{n}\right|^{2}\right)^{1 / 2}, \\
|\Im z| & =\left(\left|y_{1}\right|^{2}+\cdots+\left|y_{n}\right|^{2}\right)^{1 / 2}, \\
\langle z, t\rangle & =z_{1} t_{1}+\cdots+z_{n} t_{n},
\end{aligned}
$$

will be used for any $t \in \mathbb{R}^{n}$ and $\lambda \in \mathbb{C}^{n}$. Let $A$ denote positive constants, it may be different at each occurrence. The symbol $D(a, t)$ is used to denote the disk $|z-a|<t$. The letter A denotes positive constants and it may be different at each occurrence. Throughout this paper, the upper half-plane $\{z=x+i y: y>0\}$ is denoted by $\mathbb{C}_{+}$.

Section 2 is devoted to the study of completeness of root vectors for Schrödinger operators. The conjugate argument will be applied to characterize the completeness of root vectors for Schrödinger operators.

\section{Completeness of root vectors}

In this section, we obtain some complete criterion which is characterized by the spectra of Schrödinger operators.

We shall recall some background knowledge of Schrödinger operators. Following [4], we recall a few basic facts regarding the eigenvalues of a compact, linear operator $T$ in a Banach space $\mathbb{B}$. The geometric multiplicity, $m\left(\lambda_{0}, T\right)$, of an eigenvalue $\lambda_{0} \in \sigma_{p}(T)$ of $T$ is given by $m\left(\lambda_{0}, T\right):=\operatorname{dim}\left(\operatorname{ker}\left(T-\lambda_{0}\right)\right)$.

The root subspace of $T$ corresponding to $\lambda_{0} \in \sigma_{p}(T)$ is given by

$$
\mathrm{R}_{\lambda_{0}}(T)=\left\{f \in \mathbb{B} \mid\left(T-\lambda_{0}\right)^{k} f=0, \text { for some } k \in \mathbb{N}\right\} .
$$

Elements of $R_{\lambda_{0}}(T)$ are called root vectors. 
Denote by $\left\{\lambda_{j}\right\}_{j=1}^{\infty}$ the sequence of non-zero eigenvalues of $T$ and let $n_{j}$ be the algebraic multiplicity of $\lambda_{j}$. By a system of root vectors of the operators $T$, we mean a family of the form

$$
\bigcup_{j=1}^{\infty}\left\{e_{j k}\right\}_{k=1}^{n_{j}},
$$

where $\left\{e_{j k}\right\}_{k=1}^{n_{j}}$ is a basis in $R_{\lambda_{j}}(T)$.

We are particularly interested in the case where $T$ is a densely defined, closed, linear operator in $\mathbb{B}$.

Let us recall some basic knowledge on scattering solutions from [15]. Denote by $h(x, \alpha, \lambda)$ the scattering solution solves the scattering problem (1.1)

$$
h=e^{i \lambda<\alpha, x>}+A(\alpha, \beta, \lambda) \frac{e^{i \lambda r}}{r}+o\left(\frac{1}{r}\right),
$$

where $r:=|x| \rightarrow \infty, \beta:=\frac{x}{r}$, furthermore, $\alpha, \beta \in S^{2}$ are the unit vectors, $S^{2}$ is the unit sphere.

Let

$$
\begin{gathered}
\tilde{f}(\xi)=\int_{\mathbb{R}^{3}} f(x) e^{i<\xi, x>} d x, f(x)=\frac{1}{(2 \pi)^{3}} \int_{\mathbb{R}^{3}} e^{-i<\xi, x>} \tilde{f}(\xi) d x, \\
f * g:=\int_{\mathbb{R}^{3}} f(x-y) g(y) d y .
\end{gathered}
$$

Denote by

$$
f(x, y, \lambda):=\frac{e^{i \lambda|x-y|}}{4 \pi|x-y|}
$$

then the scattering solution $h=h(x, \alpha, \lambda)$ solves the integral equation,

$$
h(x, \alpha, \lambda)=e^{i \lambda<\alpha, x>}-\int_{B\left(r_{0}\right)} f(x, y, \lambda) q(y) h(y, \alpha, \lambda) d x .
$$

If

$$
v=e^{-i \lambda<\alpha, x>} h(x, \alpha, \lambda),
$$

then

$$
v=1-\int_{B\left(r_{0}\right)} F(x-y, \lambda) q(y) h(y, \alpha, \lambda) d x,
$$

where

Define $\epsilon$ by

$$
F(x-y, \lambda)=:=\frac{e^{i \lambda|x-y|-<\beta, x-y>}}{4 \pi|x-y|} .
$$

$$
v=1+\epsilon,
$$

then (2.1) can be rewritten as

$$
\epsilon(x, \alpha, \lambda)=-\int_{B\left(r_{0}\right)} F(x-y, \lambda) q(y) v(y, \alpha, \lambda) d x-G \epsilon,
$$

where

$$
\mathrm{G} \epsilon:=\int_{\mathrm{B}\left(\mathrm{r}_{0}\right)} \mathrm{F}(x-y, \lambda) \mathrm{q}(\mathrm{y}) \epsilon(y, \alpha, \lambda) \mathrm{d} x .
$$

We also need some results on analytic functions. We will make use of the following result from [6].

Lemma 2.1. Let $\Lambda=\left\{\lambda_{n}\right\}$ be a sequence of complex numbers satisfying

$$
0<\sup \left\{\left|\arg \lambda_{n}\right|\right\}<\pi,
$$

and

$$
\inf \left\{|| \lambda_{n}|-| \lambda_{n-1}||\right\}>0,
$$

furthermore, let $\mathrm{m}$ be a nonnegative integer. An analytic function $\mathrm{f}(\mathrm{z})$ in the upper half-plane $\mathbb{C}_{+}$vanishing exactly 
on $\Lambda=\left\{\lambda_{n}\right\}$ can be decomposed as

$$
\mathrm{f}(z)=\mathrm{B}(z) \mathrm{g}(z),
$$

where $\mathrm{g}(z)$ is zero free, the function $\mathrm{B}(z)$ is the Blaschke product

$$
\mathrm{B}(z)=\left(\frac{z-i}{z+i}\right)^{m} \prod_{n=1}^{\infty} \frac{\left|\lambda_{n}^{2}+1\right|}{\lambda_{n}^{2}+1} \frac{z-\lambda_{n}}{z-\overline{\lambda_{n}}},
$$

if and only if

$$
\sum_{n=1}^{\infty} \frac{\mathfrak{I} \lambda_{n}}{1+\left|\lambda_{n}\right|^{2}}<\infty
$$

and there exists a harmonic function $\mathrm{u}(z)$ such that

$$
\log |f(z)| \leqslant u(z)
$$

holds on $\mathbb{C}_{+}$.

Now we can prove the completeness theorems.

Theorem 2.2. Let q satisfy Assumption 1.1. If $\left\{\lambda_{n}\right\}$ is a sequence of eigenvalues of (1.1) satisfying, (2.3), (2.4) and

$$
\sum_{n=1}^{\infty} \frac{\Im \lambda_{n}}{1+\left|\lambda_{n}\right|^{2}}=\infty,
$$

then the system of root vectors of (1.1) is complete in $\mathrm{L}^{1}\left(\mathrm{~B}\left(\mathrm{r}_{0}\right)\right)$.

Proof. If $\left\{h\left(x, \lambda_{n}\right)\right\}, \lambda_{n} \in \Lambda$ is incomplete in $\mathrm{L}^{1}\left(\mathrm{~B}\left(\mathrm{r}_{0}\right)\right)$, by the Hahn-Banach Theorem, there exists a nontrivial bounded linear functional such that $\|T\|=1$ and $T\left(h\left(x, \lambda_{n}\right)\right)=0$, for $\lambda_{n} \in \Lambda$. So by the Riesz representation theorem, there exists a bounded function $\mathrm{g}$ on $\mathrm{B}\left(\mathrm{r}_{0}\right)$ satisfying

$$
\|g\|=\int_{B\left(r_{0}\right)}|g(t)| d t=\|T\|,
$$

and

$$
\mathrm{T}(\mathrm{h})=\int_{\mathrm{B}\left(\mathrm{r}_{0}\right)} \mathrm{h}(\mathrm{x}) \mathrm{g}(\mathrm{x}) \mathrm{dt}, \quad h \in \mathrm{L}^{1}\left(\mathrm{~B}\left(\mathrm{r}_{0}\right)\right) .
$$

Define

$$
f(\lambda)=\int_{B\left(r_{0}\right)} h(x, \lambda) g(x) d x,
$$

then $f(\lambda)$ is analytic in the upper half plane $\mathfrak{I} \lambda \geqslant 0$, vanishing on $\Lambda=\left\{\lambda_{n}\right\}$. Rewrite

$$
f(\lambda)=\int_{B\left(r_{0}\right)} e^{i \lambda<\zeta, x>}(1+\epsilon(x, \lambda) g(x)) d x,
$$

where $\epsilon(x, \lambda)$ is defined in (2.2), for all $\zeta \in S_{+}^{2}$. It is obvious that

$$
\sup _{\zeta \in S_{+}^{2}}\left|\int_{B\left(r_{0}\right)} e^{i \lambda<\zeta, x>} g(x) d x\right| \leqslant A e^{r_{0}|\lambda|},
$$

and

$$
\sup _{\zeta \in S_{+}^{2}}\left|\int_{B\left(r_{0}\right)}(1+\epsilon(x, \lambda)) g(x) d x\right| \leqslant A e^{r_{0}|\lambda|}
$$


Thus, for sufficiently large $|\lambda|$, we have

$$
|f(\lambda)| \leqslant A e^{r_{0}|\lambda|}
$$

Denote by

$$
f_{1}(\lambda)=\frac{f(\lambda)}{\Gamma(\lambda)}
$$

where $\Gamma(\lambda)$ is the Gamma function. It is well-known that $|\Gamma(\lambda)|=O\left(e^{|\lambda| \log |\lambda|}\right)$ holds as $|\lambda| \rightarrow \infty$. Thus, we have

$$
\left|f_{1}(\lambda)\right| \leqslant A,
$$

i.e., $f_{1}(\lambda)$ is bounded in the upper half plane $\mathfrak{I} \lambda \geqslant 0$. Applying Lemma 2.1 to $f_{1}(\lambda)$, we conclude that $f_{1}(\lambda) \equiv 0$ by (2.5), which is equivalent to $f(\lambda) \equiv 0$, then

$$
\int_{B\left(r_{0}\right)} e^{i \lambda<\zeta, x>}(1+\epsilon(x, \lambda) g(x)) d x \equiv 0 .
$$

Since the left-hand side of (2.6) depends on $\zeta$ analytically on the variety $M:=\{\langle\theta, \theta\rangle=1,<\theta, \theta\rangle=$ $\left.\theta_{1}^{2}+\theta_{2}^{2}+\theta_{3}^{2}, \theta_{j} \in \mathbb{C}, j=1,2,3\right\}$, by Lemma 2.2 in [15], equation (2.6) holds for complex $\lambda$,

$$
\lambda=u+i v, \quad v \geqslant 0 .
$$

We may derive from (2.6) the relation

$$
\left.\widetilde{\mathbf{g}}((\mathrm{u}+\mathfrak{i} v) \zeta)+\frac{1}{(2 \pi)^{3}}(\widetilde{\epsilon} * \widetilde{\mathbf{g}})((u+\mathfrak{i} v) \zeta)\right)=0, \quad \forall \zeta \in S^{2}, \quad \forall u \in \mathbb{R},
$$

where the notation $(f * g)(z)$ means that the convolution $f * g$ is calculated at the argument $z=(u+i v) \zeta$. Denote by

$$
\rho(u, v)=\sup _{\zeta \in \mathrm{S}^{2}} \int_{\mathbb{R}_{3}}|\widetilde{\epsilon}((u+i v) \zeta-s)| d s
$$

then

$$
\sup _{\zeta \in S^{2}}|\widetilde{\epsilon} * \widetilde{p}|:=\sup _{\zeta \in S^{2}}\left|\int_{\mathbb{R}_{3}} \widetilde{\epsilon}((u+\mathfrak{i} v) \zeta-s) \widetilde{\mathbf{g}}(s) d s\right| \leqslant \rho(u, v) \sup _{s \in \mathbb{R}^{3}}|\widetilde{\mathbf{g}}(s)| .
$$

From the proof of Lemma 3.1 in [15] (see page 5-7 in [15], replace $p$ by bounded $g$, one can see that (27) and (28) in [15] hold), we can derive for bounded function $\mathrm{g}$,

$$
\left.\sup _{\zeta \in S^{2}} \mid \widetilde{g}(u+i v) \zeta\right)\left|\geqslant \sup _{s \in \mathbb{R}^{3}}\right| \widetilde{g}(s) \mid, u \rightarrow \infty
$$

From the proof in [15] (see (43) and (44) there), we also have

$$
0<\rho(u, \eta(u))<1,
$$

if $\eta(u)=O(\ln u)$ is suitably chosen for $u \rightarrow \infty$. Combination of (2.7), (2.8) and (2.1) yields

$$
\widetilde{g} \equiv 0
$$

Thus, by the injectivity of the Fourier transform, we have $\mathrm{g} \equiv 0$.

We will study the completeness problem wherever the equation (1.1) has spectra on the imaginary axis. We need some more results on analytic functions from [1].

Lemma 2.3. If $\mathrm{f}(z)$ is regular for $\mathfrak{I} \geqslant 0$ and

$$
|f(z)| \leqslant e^{A|z|},
$$

and $\mathrm{f}\left(\mathrm{i} \lambda_{\mathrm{n}}\right)=0$, with $\lambda_{\mathrm{n}}>0, \lambda_{\mathrm{n}}-\lambda_{\mathrm{n}-1}>\delta>0$, then $\mathrm{f}(z) \equiv 0$, if 


$$
\limsup _{t \rightarrow \infty} t^{-2 A / \pi} \varphi(t)=\infty
$$

where

$$
\varphi(t)=\exp \left\{2 \sum_{\lambda_{n}<t} \lambda_{n}^{-1}\right\}
$$

Lemma 2.4. If $\mathrm{f}(z)$ satisfies the hypothesis of Lemma 2.3 , then $\mathrm{f}(z) \equiv 0$, if $\mathrm{A}<\pi$ and

$$
\liminf _{n \rightarrow \infty} n / \lambda_{n} \geqslant 1 .
$$

Theorem 2.5. Let q satisfy Assumption 1.1. If $\left\{\lambda_{n}\right\}$ is a sequence of eigenvalues of (1.1) satisfying (2.3), (2.4) and (2.9), where $\varphi(t)$ is defined in (2.10), furthermore, suppose that $r_{0}<\pi$, then the system of root vectors of (1.1) is complete in $\mathrm{L}^{1}\left(\mathrm{~B}\left(\mathrm{r}_{0}\right)\right)$.

Proof. We may apply the proof of Theorem 2.2. Denote by $f(z)$ the analytic function defined in the proof of Theorem 2.2, replacing Lemma 2.1 by Lemma 2.3, then the result follows from the proof of Theorem 2.2.

Theorem 2.6. Let q satisfy Assumption 1.1. If $\left\{\lambda_{n}\right\}$ is a sequence of eigenvalues of (1.1) satisfying (2.3), (2.4) and (2.11), furthermore, suppose that $\mathrm{r}_{0}<\pi$, then the system of root vectors of (1.1) is complete in $\mathrm{L}^{1}\left(\mathrm{~B}\left(\mathrm{r}_{0}\right)\right)$.

Proof. We may apply the proof of Theorem 2.2. Denote by $f(z)$ the analytic function defined in the proof of Theorem 2.2, replacing Lemma 2.1 by Lemma 2.4, then the result follows from the proof of Theorem 2.2.

\section{Acknowledgment}

The author is supported by National Natural Science Foundation of China (No.11261024).

\section{References}

[1] R. P. Boas, Jr., Entire functions, Academic Press Inc., New York, (1954). 2

[2] A. Boivin, H.-L. Zhong, Completeness of systems of complex exponentials and the Lambert W functions, Trans. Amer. Math. Soc., 359 (2007), 1829-1849. 1

[3] T. Bouhennache, Point spectrum of elliptic operators in fibered half-cylinders and the related completeness problem, Integral Equations Operator Theory, 39 (2001), 182-192. 1

[4] N. Dunford, J. Schwartz, Linear operators, Part I, General theory, With the assistance of William G. Bade and Robert G. Bartle, Reprint of the 1958 original. Wiley Classics Library, A Wiley-Interscience Publication, John Wiley \& Sons, Inc., New York, (1988). 2

[5] R. L. Frank, A. Laptev, E. H. Lieb, R. Seiringer, Lieb-Thirring inequalities for Schrödinger operators with complex-valued potentials, Lett. Math. Phys., 77 (2006), 309-316. 1

[6] J. B. Garnett, Bounded analytic functions, Pure and Applied Mathematics, Academic Press, Inc. [Harcourt Brace Jovanovich, Publishers], New York-London, (1981). 2

[7] F. Gesztesy, V. Tkachenko, A Schauder and Riesz basis criterion for non-self-adjoint Schrödinger operators with periodic and antiperiodic boundary conditions, J. Differential Equations, 253 (2012), 400-437. 1

[8] S. A. Guda, Completeness of the Floquet solutions to the problem of a solid oscillating in a fluid, Sib. Math. J., 50 (2009), 405-414. 1

[9] V. Isakov, Completeness of products of solutions and some inverse problems for PDE, J. Differential Equations, 92 (1991), 305-316. 1

[10] L. Knockaert, D. D. Zutter, On the completeness of eigenmodes in a parallel plate waveguide with a perfectly matched layer termination, IEEE Trans. Antennas. Propag., 50 (2002), 1650-1653. 1

[11] A. Laptev, O. Safronov, Eigenvalue estimates for Schrödinger operators with complex potentials, Comm. Math. Phys., 292 (2009), 29-54. 1

[12] G. V. Radzīèvksiì, Multiple completeness of the root vectors of an M. V. Keldyš pencil that is perturbed by an operator-valued function analytic in the disc, (Russian) Mat. Sb. (N.S.), 91 (1973), 310-335. 1

[13] A. G. Ramm, Completeness of the products of solutions to PDE and uniqueness theorems in inverse scattering, Inverse Problems, 3 (1987), 77-82. 1 
[14] A. G. Ramm, An inverse scattering problem with part of the fixed-energy phase shifts, Comm. Math. Phys., 207 (1999), 231-247. 1

[15] A. G. Ramm, Uniqueness of the solution to inverse scattering problem with scattering data at a fixed direction of the incident wave, J. Math. Phys., 52 (2011), 12 pages. 2, 2, 2, 2

[16] Z. Wang, H.-Y. Wu, The completeness of eigenfunctions of perturbation connected with Sturm-Liouville operators, J. Syst. Sci. Complex., 19 (2006), 527-537. 1

[17] Y. Yakubov, Completeness of elementary solutions of second order elliptic equations in a semi-infinite tube domain, Electron. J. Differential Equations, 2002 (2002), 21 pages. 1

[18] X.-D. Yang, Incompleteness of exponential system in the weighted Banach space, J. Approx. Theory, 153 (2008), 73-79. 1

[19] X.-D. Yang, Random inverse spectral problems and closed random exponential systems, Inverse Problems, 30 (2014), 12 pages. 1

[20] X.-D. Yang, J. Tu, On the completeness of the system $\left\{\mathrm{t}^{\lambda_{n}}\right\}$ in $\mathrm{C}_{0}(\mathrm{E})$, J. Math. Anal. Appl., 368 (2010), 429-437. 1 